\title{
Strange Carers: Robots as Attachment Figures and Aids to Parenting
}

\section{Dean Petters}

University of Birmingham

\section{Everett Waters}

State University of New York (Stony Brook)

\section{Felix Schönbrodt}

Humboldt University (Berlin)

\begin{abstract}
Sharkey \& Sharkey (2010) have examined recent research and developments in childcare robots and speculated on progress over the coming years by extrapolating from other ongoing robotics work. They raise ethical questions about the use of robots as part or full-time carers, focusing on issues of human rights, privacy, robot use of restraint, deception of children and accountability, and consequences for the psychological and emotional wellbeing of children. They consider the adequacy of current legislation and international ethical guidelines on the protection of children from the overuse of robot care.
\end{abstract}

The present comment focuses on the distinction between attachment as bond formation and expectations of availability and responsiveness (security) within attachment relationships. We enumerate key components of bonding and functions of carer secure base support. Our analysis has implications for design and suggests that robots are unlikely to serve effectively as sole carers in the near or far-near future. Even with robots as part-time carers, attachment-like bonds and related learning would likely focus on human carers. Similarly, although infants and children would certainly build expectations regarding the availability and responsiveness of robot carers, the quality of human care would probably be the determining influence on later development and competence. Notwithstanding their limitations of robots as attachment figures they have considerable potential to extend parental care and enrich exploration in infancy and childhood. The Sharkey's paper and further consideration of robots as carers for infants, children, older adults, and individuals with special needs are of theoretical and practical interest and should be pursued.

Qharkey \& Sharkey (2010) have written a most $\checkmark$ interesting paper, raising interesting ethical and practical issues regarding robots as sole and parttime carers. What competencies can robots achieve in the near term? Can we experience social relatedness with them (and they with us)? Can robots care (in the sense of nurture) for us? Are their risks in deploying robots as carers for the young? Can we care (in the affective sense) for them?

As the Sharkey's note (p. 11) some definitional difficulties attend the term attachment. This is not to say that attachment is beyond definition, only that it often goes undefined. How one approaches the roles of robots as carers begins with how one conceptualizes attachment. Are we referring to a specific mode of relating or to social relatedness in general? And how do attachment relationships differ from one to another?

\section{Attachment}

Bowlby (e.g., 1969), Ainsworth, Blehar, Waters, \& Wall (1978), and Sroufe \& Waters (1977) have provide a definitional framework that defines attachment as a significant relationship distinct from friendship and other social ties. They highlight as the hallmarks of attachment (a) preference, (b) familiarity , (c) relative uniqueness, (d) identity, (d) use as a secure base, and (e) grief and mourning in response to loss. 
Attachment figures are preferred to others, especially in the face of uncertainty or danger. They are familiar in the sense that one has a history of interaction with them upon which expectations about their availability, responsiveness, and competence are built. Bowlby often spoke of attachment as monotropic, tending to be focused on one or a very few figures. Current thinking favors relative uniqueness; that is, a person might view more than one or two individuals as attachment figures but the number would be far fewer than they might know as friends, neighbors, acquaintances, colleagues, or well-wishers. In addition to being bounded in number, each has a distinct identity in that they are experienced as individuals rather than as members of an indistinct class; they are not strictly interchangeable and expectations about availability and responsiveness can differ from one to the other. Most importantly, an attachment figure is someone who is used as a secure base from which to explore and as a haven of safety in retreat. As a secure base for exploration, attachment figures play an important role in developing knowledge, experience, and competencies that are important to later development and adaptation. As a haven of safety, they play an important role in physical security and affect regulation. And finally, attachment is associated with a distinctive response to loss that marks such relationships as uniquely significant and deeply integrated into a person's understanding of their world and their self.

This perspective reflects a great deal of ethological observation, empirical research, and theoretical analysis and has proven useful in translating theory into practice. Moreover, definitions play an important implication for the kinds of competencies we would look for in a robot carer.

\section{Security in Attachment Relationships}

Informally, the term secure attachment suggests a bond that is tight or strong. This was the sense in psychoanalytic and classical learning theories. With the decline of drive theories, the notion that attachments differ primarily in strength has fallen into disuse. Instead, individual differences are conceptualized in terms of the cognitive and behavioral facets of secure base use and support. To be secure in an attachment relationship means (a) to be confident in an attachment figure's availability, responsiveness, and competence - the expectation of that person being "always there for me", and (b) as a result to explore confidently from the attachment figure as a secure base and to find ready comfort in proximity and contact as needed. The difference between attachment as intensity versus quality is nicely captured in the German translation of security as sicherheit (certainty or confidence). Primarily implicit, pattern based, and automatic, the cognitive and behavioral components of attachment have strong emotional associations and induce strong emotional responses when confirmed, violated, impeded, or interrupted.

Concepts like presence versus absence and degree of consolidation have proven difficult to operationalize. Instead, since the 1970's researchers have used naturalistic observations, the Ainsworth Strange Situation laboratory procedure, and the Berkeley Adult Attachment Interview to assess the cognitive and behavioral components of security in attachment relationships in developmental, clinical, and cross-cultural studies. Much of this work is reviewed in the recent Handbook of Attachment (Cassidy \& Shaver, 2008).

Observational research has demonstrated that the expectations of an attachment figure's availability and responsiveness we call attachment security or insecurity (a) arise from actual experience, (b) tend to be stable into childhood and early adulthood, (c) are open to revision in light of further experience, (d) can differ from one relationship to another, and (e) can provide a prototype around which are constructed initial expectations in adult relationships and initial goals in parenting (e.g., Sroufe, Egeland, Carlson, \& Collins, 2005; Grossmann, Grossmann, \& Waters, 2005).

\section{Risks Attending Attachment to Robot Carers}

The distinction between attachment (bondedness) and security-insecurity (expectations about the availability and responsiveness of an attachment figure) points to different mechanisms and different risks.

\section{Imprinting}

Aside from work on species identification and imprinting in non-human species, we know very little about the role early experience plays in activating the capacity to form lasting bonds or any impact early experience might have on later social responsiveness. However, Rutter (e.g. 1999) has concluded from a detailed review of clinical research that lack of opportunity to form attachment 
(usually due to early chronic illness, institutional care, or multiple foster placements) is a significant factor in later psychopathology. The question then is whether bonding to a robot is better than not bonding at all, and whether it provides sufficient foundations for later social learning.

As the Sharkey's point out, endowing a robot with even minimal caregiving competence will be a challenge. Asimov's prescription that a robot may not injure a human being or, through inaction, allow a human being to come to harm implies considerable expertise in what is necessary and tolerable moment to moment, and over significant periods of development. Few, if any, adults begin their parenting careers with such expertise. They depend instead on a great deal of trial and error, feedback from the child, problem solving, error correction and compensatory behavior, and observational learning. Much of our success as parents ultimately depends on living in communities of helpful more experienced carers and on the fact that skills not successfully prepared or instilled by primary carers can be "backed up" by learning in the peer group and in other social contexts. Even human carers would likely fail if they undertook child rearing in such an impoverished environment. The plausibility then of robots as sole carers depends not only on endowing them with caregiving skills but on their potential for social learning.

The challenges to robots as sole carers increase dramatically as the child grows older. As the child's cognitive skills increase, the attachment figure's contributions become increasingly abstract and difficult to implement in software. Even attachment researchers often assume that the function of attachment figures is primarily to insure safety and support social-emotional development. But a major function of attachment is to support the growth of competence and independence (Waters, 2002). Secure base figures are not simply someone to run to in an emergency. They play an important role in making the child feel comfortable to explore new environments, practice and consolidate skills, gain practical knowledge about the self and the physical and social world, and build conceptual tools that support adaptive independent behavior. To mention just a few components of good secure base support, the secure base figure enriches exploration by:

- guiding attention

- giving explanations
- modelling positive affect

- modelling caution and reassurance

- anticipating, pointing out or clearing away obstacles to safe productive exploration

- helping resolve problems so that exploration is maintained and profitable

- making sure the child does not overlook possibilities for deeper exploration

- helping the child construct interpretations - e. g., was this experience typical or an outlier?

- providing plausible information about mechanisms

- listening to the child's explanations and refining or extending them

Even if robots could perform basic maintenance of young infants, the skills required of a competent attachment figure become almost impossibly abstract. We simply don't know enough about the mechanisms of bonding and later sociability to know whether bonding to a robot carer as a primary attachment figure would be damaging. In our estimate robots will not be judged competent to the task in the near term.

\section{Insecurity}

A second concern has to do with robots' competence as supplementary carers - can they support confident expectations of availability and responsiveness (secure attachment) or would they necessarily engender insecure attachments and unacceptable risks to later adjustment. Let us assume that robots would most likely be serving as semiautonomous carers performing maintenance, supervision, and enrichment activities for intervals and in specific contexts. This would be analogous to the doctor-nurse relationship; the doctor (parent) is responsible for the overall plan of care and for specific decisions and procedures; the nurse (robot) acts on specific instructions, exercises a considerable range of autonomy in other respects, and has a clear protocol for alerting and returning control to the doctor. In such an arrangement, it seems likely that only the tie to the primary human carer would meet the above criteria for attachment. Familiarity, preference, and some expectations about availability and responsiveness would be possible; perhaps even some basic retreat to the robot when distressed (if only to activate a call to the parent). Uniqueness, identity, complex secure base use, and 
mourning in response to loss seem less plausible. It would seem then that experience with the human carer, expectations about its availability and responsiveness, and experience using it as a secure base for exploration, i.e. the quality of the relationship to the human carer, would determine whether the child realized the benefits of a secure caregiving relationship. Obviously, the child's view of its parent as available, responsive, always there for me, could be enhanced or compromised by how the parent deployed the robot carer. The Sharkey's cite examples of parents whose use of robot carers seems quite selfish and neglecting. Would such parents engender confidence and security in their own interactions with the child? Would an older child not sense that the parents are unavailable and selfish? In all likelihood this, not the quality of care provided by the robot, would play the greater role in shaping later character and adjustment.

\section{Conclusion}

Isaac Asimov's (1940) short story 'Strange Playfellow' describes how a young girl called Gloria forms a strong attachment bond towards Robbie, her robotic carer. Even with the nearly 70 years of technological development since 'Strange Playfellow' was published, robots with the capability of Robbie are still an unrealisable fiction. In highlighting this reality gap between possible future aspirations for robotic carers and their present potential, Sharkey and Sharkey have stimulated interaction between robotics and attachment study, and helped clarify what are worthwhile and achievable aims for current research. Considering how and why currently achievable robots might fail as carers for human infants is to the advantage of both robotics and attachment theory. This is because to answer these questions involves firstly considering the developmental requirements for, and functions of, attachment relationships, and then considering how human carers fulfill these requirements and functions.

Although robots will not be effective sole carers of infants in the near term, they may find useful roles extending the range and quality of care that humans can provide, and enriching childhood exploration and in providing care for older adults and individuals with special needs. We agree that each of these applications has ethical dimensions even if the stakes for robotic carers are less high, and may avoid the hazards which Sharkey and Sharkey set out in their interesting analysis. Many existing examples of human-robot interaction involve only short term interactions. Sharkey and Sharkey have taken a useful step toward exploring the full range of robot human interactions and promoting interdisciplinary collaboration.

\section{References}

Ainsworth, M., Blehar, M., Waters, E., Wall, S., (1978) Patterns of Attachment: a psychological study of the strange situation. Erlbaum, Hillsdale, NJ, 1978.

Asimov, I. (1940). Strange Playfellow, Super Science Stories, September, pp. 67-77.

Bowlby, J. (1969). Attachment and Loss: volume 1 Attachment. Basic books, New York.

Cassidy, J. \& Shaver, P. (2008). Handbook of attachment: Theory, research, and clinical applications (2nd Ed). New York: Guilford.

Grossman, K.E., Grossman, K. \& Waters, E., (2005). Attachment from Infancy to Adulthood. New York: Guilford Publications.

Rutter, M. (1999). Maternal deprivation reassessed. London: Penguin Books UK.

Sharkey, N. \& Sharkey, A. (2010). The crying shame of robot nannies: an ethical appraisal. Interaction Studies: Social Behaviour and Communication in Biological and Artificial Systems (in press).

Sroufe, L. A., Egeland, B., Carlson, E., \& Collins, W. A. (2005) The Development of the Person: The Minnesota Study of Risk and Adaptation from Birth to Adulthood. New York: Guilford Publications.

Sroufe, L. A., \& Waters, E. (1977). Attachment as an organizational construct. Child Development, 48, 1184-1199.

Waters, E. (2002). Live long and prosper. Essay retrieved online October, 20, 2009. http:// www.psychology.sunysb.edu/attachment/ gallery/live_long/live_long.html 